\title{
$A b$ Initio Study of the Ferromagnetic Response, Local Structure, and Hyperfine Properties of Fe-Doped $\mathrm{SnO}_{2}$
}

\author{
A. M. Mudarra Navarro, ${ }^{*}{ }^{\dagger}$ C. E. Rodríguez Torres, ${ }^{\dagger}$ A. F. Cabrera, ${ }^{\dagger}$ M. Weissmann, ${ }^{\ddagger}$ K. Nomura, ${ }^{\S}$ \\ and L. A. Errico ${ }^{\dagger, \|}$ \\ ${ }^{\dagger}$ Departamento de Física - Facultad de Ciencias Exactas, UNLP, Instituto de Física La Plata, CCT-La Plata, CONICET, CC 67, 1900 \\ La Plata, Argentina

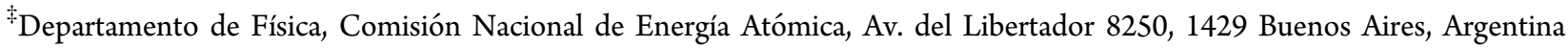 \\ ${ }^{\S}$ School of Engineering, The University of Tokyo, 7-3-1 Hongo, Bunkyo-ku, Tokyo 113-8656, Japan \\ "Universidad Nacional del Noroeste de la Pcia. de Buenos Aires (UNNOBA), Monteagudo 2772, Pergamino, CP 2700 Buenos Aires, \\ Argentina
}

ABSTRACT: We present here an ab initio study of the structural, magnetic, and hyperfine properties of Fe-doped rutile $\mathrm{SnO}_{2}$ for different concentrations and distributions of the $\mathrm{Fe}$ atoms and oxygen vacancies in the $\mathrm{SnO}_{2}$ host. The calculated results are compared with experimental ones obtained by Mössbauer spectroscopy and X-ray absorption techniques. This comparison enables us to characterize the local structure around $\mathrm{Fe}$ atoms and to identify the different hyperfine interactions that are observed in samples prepared by different methods. It is concluded that oxygen vacancies are

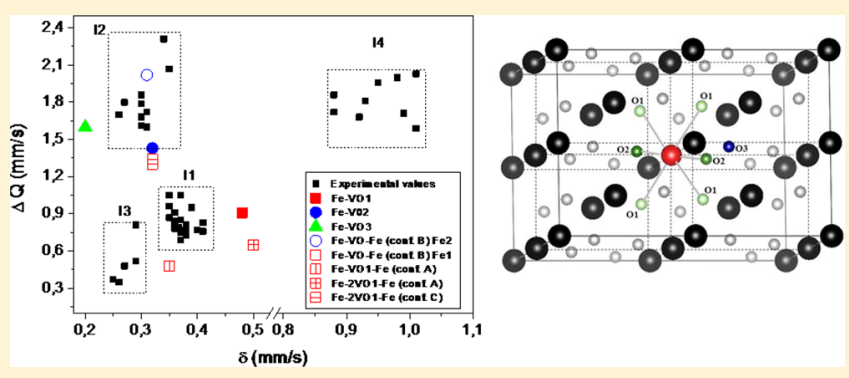
fundamental for the ferromagnetic response of Fe-doped $\mathrm{SnO}_{2}$. The $a b$ initio calculations show that two $\mathrm{Fe}$ ions sharing an oxygen vacancy are coupled ferromagnetically, forming a bound magnetic polaron (BMP), and that two neighbor BMPs are aligned antiparallel to each other. Electron doping plays a fundamental role mediating the magnetic coupling between the BMP inducing ferromagnetic alignment between the BMPs.

\section{INTRODUCTION}

Tin dioxide $\left(\mathrm{SnO}_{2}\right)$ is an n-type broad-band gap semiconducting oxide with outstanding optical, electrical, and mechanical properties. It is a versatile material especially attractive for gas sensor applications. ${ }^{1,2}$ Doping $\mathrm{SnO}_{2}$ with transition metal ions is one of the most promising ways to extend light absorption into the visible range, thereby extending its photocatalytic response (see ref 3 and references therein).

$\mathrm{SnO}_{2}$ doped with transition metal ions is also of significant interest in the emerging area of spintronics. The goal of this research field is to extend the properties and applications of established electronic devices by making use of the spin of electrons in addition to their charge to convey information. Such spin-polarized electronic devices could be much smaller, consume less energy, and be more efficient than the current electronic devices, which are based on electron charge only. In addition to its potential utility, the study of spin-polarized transport is revealing a new fundamental physics. Transitionmetal-doped $\mathrm{SnO}_{2}$ systems have been investigated extensively, following reports on the discovery of room temperature ferromagnetism. $^{4-10}$ However, contradictory experimental as well as theoretical results were obtained, and it is uncertain whether the ferromagnetic signal (FM) found is intrinsic or comes from extrinsic magnetic phases. Additionally, different experimental and theoretical works suggest that oxygen vacancies play an important role in the ferromagnetism of transition-metal-doped $\mathrm{SnO}_{2}{ }^{4-6,9,11}$ and even in thin films of pure $\mathrm{SnO}_{2}{ }^{12,13}$ and otherwise nonmagnetic oxides (see ref 14 and references therein). One of the mechanisms proposed to explain the observed ferromagnetism suggests that only small regions of the sample that contain defects (such as grain boundaries) become ferromagnetic. The defects, for example oxygen vacancies, produce an impurity band inside the oxide band gap, and a Stoner-type criterion could be used to predict the induced magnetism. The dopants would contribute to this picture but not as the determining factor. ${ }^{15,16}$

The interpretation of the experimental results were usually based on conjectures, and relatively little attention was given to the local structure around the dopants. However, the knowledge of the local environment of the dopant may be essential to understand the mechanisms giving rise to magnetic order in these compounds.

In this work we present the results of a theoretical investigation of Fe-doped rutile $\mathrm{SnO}_{2}$ considering different locations (substitutional and interstitial sites), concentrations, and distributions of the $\mathrm{Fe}$ atoms in the host lattice. The effect

Received: December 16, 2014

Revised: February 18, 2015

Published: February 20, 2015 
Table 1. Experimental Results Obtained in Mössbauer Spectroscopy Experiments for Fe-Doped $\mathrm{SnO}_{2}{ }^{a}$

\begin{tabular}{|c|c|c|c|c|}
\hline \multirow[b]{2}{*}{ sample } & \multirow[b]{2}{*}{ method of preparation } & \multicolumn{2}{|c|}{ Mössbauer } & \multirow[b]{2}{*}{ ref } \\
\hline & & $I S(\mathrm{~mm} / \mathrm{s})$ & $|\Delta Q|(\mathrm{mm} / \mathrm{s})$ & \\
\hline $\mathrm{Sn}_{0.86} \mathrm{Fe}_{0.14} \mathrm{O}_{2}$ & hydrothermally synthesized & 0.38 & 0.77 & 23 \\
\hline \multirow[t]{2}{*}{$\mathrm{Sn}_{0.90} \mathrm{Fe}_{0.1} \mathrm{O}_{2}$} & sol-gel $\left({ }^{57} \mathrm{Fe}\right.$ enriched $)$ & $0.36-0.37$ & $0.69-0.83$ & 8 \\
\hline & & $0.41-0.46$ & $1.56-2.07$ & \\
\hline $\mathrm{Sn}_{0.90} \mathrm{Fe}_{0.1} \mathrm{O}_{2}$ & simple hydrothermal & 0.38 & 0.73 & 26 \\
\hline $\mathrm{Sn}_{0.95} \mathrm{Fe}_{0.05} \mathrm{O}_{2}$ & pulsed-laser deposition & 0.29 & 0.81 & 6 \\
\hline $\mathrm{Sn}_{0.95} \mathrm{Fe}_{0.05} \mathrm{O}_{2}$ & chemically synthesized & 0.37 & 0.85 & 4 \\
\hline \multirow[t]{2}{*}{$\mathrm{Sn}_{0.95} \mathrm{Fe}_{0.05} \mathrm{O}_{2}$} & ball-milling & 0.41 & 0.83 & 27 \\
\hline & & 0.93 & 0.92 & \\
\hline \multirow[t]{2}{*}{$\mathrm{Sn}_{1-x-y} \mathrm{Fe}_{x} \mathrm{Sb}_{y} \mathrm{O}_{2-\delta}$} & sol-gel & $0.26-0.29$ & $1.60-1.8$ & 28 \\
\hline & & $0.29-0.34$ & $0.7-0.81$ & \\
\hline \multirow[t]{2}{*}{$\mathrm{Sn}_{0.90} \mathrm{Fe}_{0.1} \mathrm{O}_{2}\left({ }^{57} \mathrm{Fe} 1 \times 10^{17} / \mathrm{cm}^{2}\right)$} & ${ }^{57} \mathrm{Fe}$ implanted $\mathrm{SnO}_{2}$ at room temperature & $0.35-0.39$ & $0.95-1.05$ & 29 \\
\hline & & $0.95-1.01$ & $1.96-2.03$ & \\
\hline \multirow[t]{2}{*}{$\mathrm{SnO}_{2}$ film $\left({ }^{57} \mathrm{Fe}: 1 \times 10^{17} / \mathrm{cm}^{2}, 5 \times 10^{16} / \mathrm{cm}^{2}\right)$} & ${ }^{57} \mathrm{Fe}$ implanted $\mathrm{SnO}_{2}$ at $573 \mathrm{~K}$ & $0.25-0.29$ & $0.35-0.52$ & 29 \\
\hline & & $0.99-1.15$ & $1.43-1.71$ & \\
\hline \multirow[t]{2}{*}{$\mathrm{SnO}_{2}$ film $\left({ }^{57} \mathrm{Fe}\right.$ concentration $\sim 5 \times 10^{-4}$ at. $\left.\%\right)$} & ${ }^{57} \mathrm{Mn}$ implanted $\mathrm{SnO}_{2}$ & 0.48 & 0.91 & 30 \\
\hline & & 0.885 & 2.20 & \\
\hline
\end{tabular}

of oxygen vacancies on the hyperfine parameters, magnetic moments, and the magnetic alignments for Fe-doped $\mathrm{SnO}_{2}$ is studied as a function of oxygen vacancy concentration and dopant/oxygen vacancy distribution. The comparison with experimental studies enables us to characterize the local structure around the iron atoms in rutile unambiguously and supports the conclusion that oxygen vacancies induced by doping with metallic atoms are fundamental for the ferromagnetic response of these systems, explaining the diversity of magnetic moments observed experimentally in samples grown under different conditions.

This paper is organized as follows. The next section describes the details of the computational method. In section 3, experimental results reported by other authors as well as our own results, concerning structure and Mössbauer hyperfine parameters (focusing on those that could be compared with calculated ones), are reviewed. Section 4 contains the theoretical results and the comparison with experimental ones. In section 5 a long-range ferromagnetic order is considered.

\section{COMPUTATIONAL DETAILS}

The unit cell of rutile $\mathrm{SnO}_{2}$ contains two $\mathrm{Sn}$ atoms at the positions $(0,0,0)$ and $(1 / 2,1 / 2,1 / 2)$ and four oxygen atoms at the positions $\pm(u, u, 0)$ and $\pm(1 / 2+u, 1 / 2+u, 1 / 2)$ with $u=$ 0.3056. The lattice parameters are $a=b=4.73741 \AA$ and $c=$ $3.18641 \AA .{ }^{17}$ In this structure, the $\mathrm{Sn}$ atoms are surrounded by a slightly distorted octahedron of oxygen nearest neighbors (ONN) atoms, with a rectangular basal plane having four oxygen atoms at a distance $2.05 \AA$ (O1) and two vertex atoms at $2.06 \AA$ (O2) from $\mathrm{Sn}$. For the oxygen atoms, there is also only one crystallographic site, and each oxygen atom is surrounded by three $\mathrm{Sn}$ atoms.

We have simulated the doped systems considering periodically repeated supercells (SCs), in which some $\mathrm{Sn}$ atoms are replaced by $\mathrm{Fe}$ atoms in the rutile $\mathrm{SnO}_{2}$ host with and without oxygen vacancies. The SC considered here consists of eight unit cells of rutile $\mathrm{SnO}_{2}$. The resulting 48-atom SC has dimensions $a^{\prime}=2 a=b^{\prime}=2 b=9.47482 \AA$ and $c^{\prime}=2 c=6.37282 \AA$. If only one $\mathrm{Sn}$ atom is replaced, the composition is $\mathrm{Sn}_{15} \mathrm{FeO}_{32}$, which represents about 6 at. \% of Fe doping. SCs of dimensions $a^{\prime}=$ $b^{\prime}=a, c^{\prime}=2 c\left(25\right.$ at. \%); $a^{\prime}=b^{\prime}=2 a, c^{\prime}=3 c\left(4\right.$ at. \%), and $a^{\prime}=$ $b^{\prime}=3 a, c^{\prime}=2 c(3$ at. \%) were also considered.

The $a b$ initio spin-polarized electronic-structure calculations were performed using the $a b$ initio code Wien $2 \mathrm{~K},{ }^{18}$ which is an implementation of the full-potential augmented plane waves plus local orbitals (APW+lo) method. ${ }^{19,20}$ The parameter $R K_{\text {MAX }}$, which controls the size of the basis set, was set to 7.0 ( $R$ is the smallest muffin tin radius and $K_{M A X}$ the largest wavenumber of the basis set). Integration in the reciprocal space was performed using the tetrahedron method taking up to $100 k$-points in the first Brillouin zone. Atomic displacements around the iron atoms and oxygen vacancies have been obtained in the standard way described in ref 21. Exchange and correlation effects were treated within density-functional theory using the local spin density approximation (LSDA). ${ }^{22}$ The LDA $+U^{23}$ formalism with the same value of $U(5.4 \mathrm{eV})$ for the $3 \mathrm{~d}$ states of $\mathrm{Ti}$ and $\mathrm{Fe}$ was also applied for some selected systems. Since none of the resulting hyperfine properties and equilibrium (lowest energy) structures obtained using LDA $+U$ differs qualitatively from those obtained with LSDA, we will center our discussion in the results obtained with LSDA.

To check the accuracy of the calculated results, we have performed several additional calculations. For selected systems, we increased the basis from $R K_{M A X}=5.0$ to $R K_{\text {MAX }}=8.0$ and increased the number of $k$-points to 200 . It is found that energy differences, magnetic moments, quadrupole splittings, isomer shifts, and interatomic distances could be obtained with adequate precision using the parameters mentioned in the previous paragraph.

\section{REVIEW OF EXPERIMENTAL RESULTS}

Before discussing the results of our calculations, we will review in this section the experimental results reported by other authors as well as our own, concerning structural and Mössbauer hyperfine parameters at the $\mathrm{Fe}$ sites in $\mathrm{Fe}$-doped $\mathrm{SnO}_{2}$, while focusing on those that could be compared with the calculations.

Spectroscopy based on the Mössbauer effect is an experimental technique enabling simple comparison with $a b$ 
initio calculations. In this work we compare the experimental quadrupole splittings $(\Delta Q)$ and isomer shifts (IS) with the calculated ones. The first one is a "fingerprint" of the local symmetry of the charge distribution around the probe nucleus ( $\mathrm{Fe}$ in the present case), and the second one provides information on the charge density of s-electrons at the probe nucleus and also on the local chemical bond. ${ }^{24}$ Several previous papers have used Mössbauer spectroscopy to characterize $\mathrm{Fe}$ doped rutile $\mathrm{SnO}_{2},{ }^{4,6,7,25-30}$ and most of them have only detected paramagnetic interactions at room temperature (RT). The reported $\Delta Q$ and $I S$ values are shown in Table 1 and Figure 1 . The hyperfine interactions can be grouped mainly in

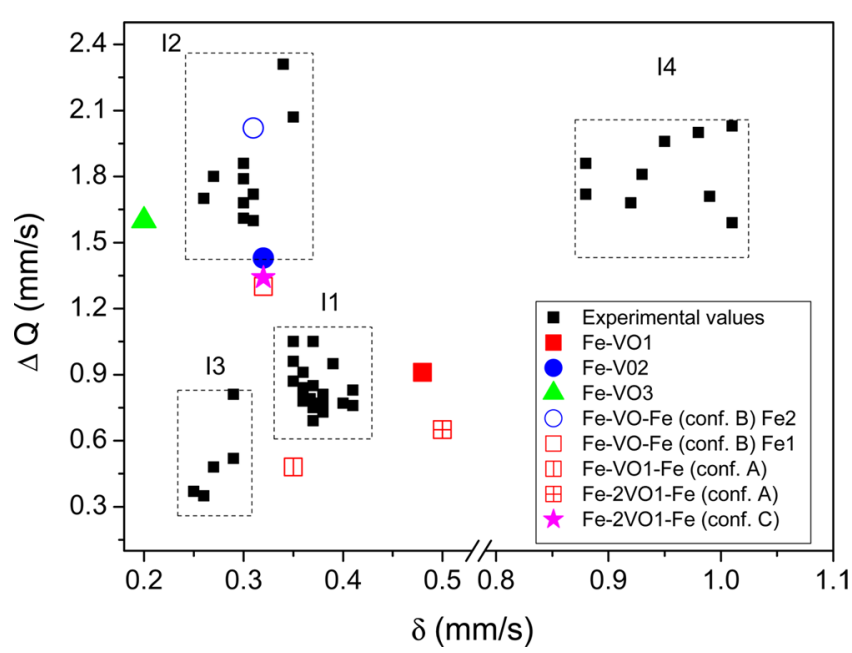

Figure 1. Relationship between IS and $\Delta Q$ obtained from experimental results and $a b$ initio calculations. The experimentally obtained interactions were classified in four classes (I1, I2, I3, and I4) according to the $I S$ and $\Delta Q$ values (see text).

four classes (I1, I2, I3, and I4), depending on the ranges of $\Delta Q$ and IS values. I1, I2, and $\mathrm{I} 3$ correspond to $\mathrm{Fe}$ in the $3^{+}$ oxidation state and $\mathrm{I} 4$ to $\mathrm{Fe}$ in to $2^{+}$oxidation state. ${ }^{31} \mathrm{I} 1$ and $\mathrm{I} 2$ are present in almost all reported studies. In all cases, I1 has a higher relative abundance than I2. I3 appears in few cases (implanted samples and thin films), and I4 is observed only in samples obtained by nonequilibrium preparation methods such as high-energy ball milling and ion implantation of Fe.

\section{THEORETICAL RESULTS}

All calculations were performed for bulk three-dimensional systems. Our spin-polarized and non-spin-polarized $a b$ initio calculations showed that the spin-polarized solutions have lower energies than non-spin-polarized ones. Therefore, we will report only the spin-polarized cases.
4.1. System with One Substitutional Fe Atom $\left(\mathrm{Sn}_{15} \mathrm{FeO}_{32}\right)$. To begin, we replaced one $\mathrm{Sn}$ atom of the rutile structure by a $\mathrm{Fe}$ one, and we let all atoms to move into the relaxed position. It is found that the presence of substitutional $\mathrm{Fe}$ in the rutile $\mathrm{SnO}_{2}$ host produces an almost isotropic structural distortion, shortening the $\mathrm{Fe}-\mathrm{ONN}$ bond lengths. As can be seen in Table 2, the $\mathrm{Fe}-\mathrm{ONN}$ bond lengths are reduced by $0.14 \AA$ with respect to the $\mathrm{Sn}-\mathrm{ONN}$ ones.

Table 2 also shows the calculated magnetic moments. It is found that they are almost independent of the local structural distortions and of the $\mathrm{Fe}$ concentration. Spin polarization occurs mainly at the Fe sites, but the ONN can be polarized up to $0.06 \mu_{\mathrm{B}} / \mathrm{ONN}$. The IS (Table 2) is referred to $\alpha$-Fe, and in order to evaluate it, we used a calibration constant $\alpha=0.25 a_{0}{ }^{3}$ $\mathrm{mm} / \mathrm{s}^{32,33}$ The value of the nuclear quadrupole moment $Q$ of the ${ }^{57} \mathrm{Fe}$ nucleus ${ }^{34}$ used to calculate the $\Delta Q$ was $-0.16 \mathrm{~b}$.

We found that the average $\mathrm{Fe}-\mathrm{ONN}$ relaxed bond lengths (1.913 A) predicted by the calculations are in poor agreement with the results of extended X-ray absorption fine structure (EXAFS, $\mathrm{Fe}-\mathrm{ONN}$ bond lengths larger than $1.98 \AA$; see ref 26). Furthermore, our $a b$ initio calculations predict an $I S$ in the order of $0.1 \mathrm{~mm} / \mathrm{s}$ (Table 2), which is far from the experimental results (Table 1 ), and the calculated $\Delta Q$ does not agree with the results obtained from the experiments (Table 1 and Figure 1). Therefore, we conclude that a substitutional doping model cannot account for the Mössbauer and EXAFS experimental results. Also, the EXAFS experiments show that the Fe coordination number is smaller than 6 (on the order of 4.8 , see ref 26). For the above reasons we will explore a second scenario that includes the formation of oxygen vacancies along with substitutional $\mathrm{Fe}$ doping.

4.2. Study of the System $\mathrm{Sn}_{15} \mathrm{FeO}_{31}$ (One Oxygen Vacancy + Substitutional Fe). We studied the oxygendeficient system $\mathrm{Sn}_{15} \mathrm{FeO}_{31}$ considering three possibilities for removing an oxygen atom from the SC: (i) from the $\mathrm{Fe}$ contained octahedron removing $\mathrm{O} 1$ atom; (ii) from the $\mathrm{Fe}$ contained octahedron removing an $\mathrm{O} 2$ atom; (iii) from an Sncontained octahedron removing $\mathrm{O} 3$ atom (see Figure 2). In all cases, it is found that the structural relaxations induced by the oxygen vacancies are larger than those produced by only doping (see Table 2). Comparing the total energies of the three cases (and even larger $\mathrm{Fe}$-oxygen vacancies separations), it is found that the oxygen vacancies near the $\mathrm{Fe}$ dopants (sites $\mathrm{O} 1$ and O2) are more stable than those near Sn atoms and that the vacancy at $\mathrm{O} 1$ site is more stable than the vacancy at $\mathrm{O} 2$ site. Similar results have been obtained in the case of Fe doping in rutile $\mathrm{TiO}_{2} \cdot{ }^{14,35-37}$ One important result is that the energy required to form an oxygen vacancy is smaller in the Fe-doped $\mathrm{SnO}_{2}$ system than in the pure one. This reduction is more important if the vacancies are close to the Fe ions. Therefore,

Table 2. Predicted Average Equilibrium Bond Lengths $\langle d\rangle$ between the Fe Atom and the Neighbor Oxygen Atoms, Magnetic Moments in the Muffin-Tin Sphere of Fe $\left(\mu^{\mathrm{imp}}\right)$ and in the Supercell $\left(\mu^{\mathrm{SC}}\right)$, and Hyperfine Parameters $\Delta Q$ and IS at the Fe Sites $^{a}$

\begin{tabular}{|c|c|c|c|c|c|}
\hline & \multirow[b]{2}{*}{$\mathrm{SnO}_{2}$} & \multirow[b]{2}{*}{$\mathrm{Sn}_{15} \mathrm{FeO}_{32}$} & \multicolumn{3}{|c|}{$\mathrm{Sn}_{15} \mathrm{FeO}_{31}$} \\
\hline & & & vacancy at site $\mathrm{O} 1$ & vacancy at site $\mathrm{O} 2$ & vacancy at site $\mathrm{O} 3$ \\
\hline$\langle d\rangle$ & 2.057 & 1.913 & 1.967 & 1.912 & 1.918 \\
\hline$\mu^{\mathrm{imp}} / \mu^{\mathrm{SC}}$ & & $1.63 / 2.00$ & $3.62 / 4.54$ & $2.42 / 2.99$ & $1.44 / 1.88$ \\
\hline$|\Delta Q|(\mathrm{mm} / \mathrm{s})$ & & 2.43 & 0.91 & 1.43 & 1.60 \\
\hline IS $(\mathrm{mm} / \mathrm{s})$ & & +0.13 & +0.48 & +0.32 & +0.20 \\
\hline
\end{tabular}

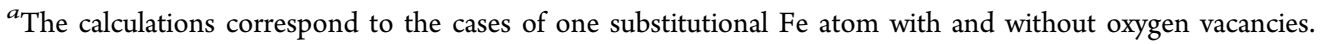




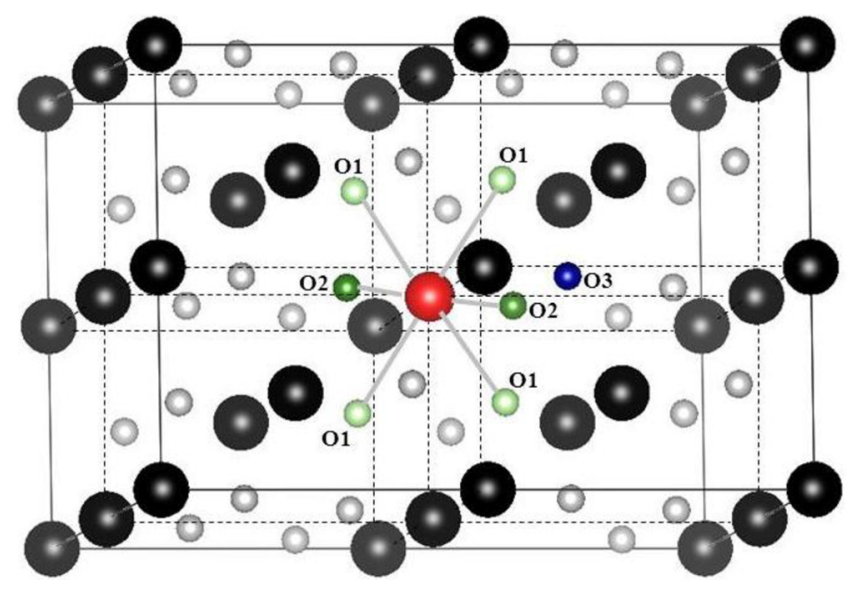

Figure 2. Eight unit cells that form the $2 \times 2 \times 2$ supercell of Fedoped rutile $\mathrm{SnO}_{2}$. The different types of atoms and Fe oxygen nearest neighbors are indicated ( $\mathrm{Sn}$ : black ball; Fe: red ball; oxygen types $\mathrm{O} 1$ and O2: green balls; and oxygen type O3: blue ball).

we predict that $\mathrm{Fe}$-doped $\mathrm{SnO}_{2}$ systems will have more vacancies than the undoped ones and that oxygen vacancies will tend to be close to the $\mathrm{Fe}$ atoms.

Table 2 shows that the presence of an oxygen vacancy close to the Fe atoms increases the magnetic moment of the SC. This magnetic moment is originated mostly by the polarization of the $\mathrm{Fe}$ atoms. Regarding the hyperfine parameters at the $\mathrm{Fe}$ sites in $\mathrm{Sn}_{15} \mathrm{FeO}_{31}$, an oxygen vacancy located at O1, O2, or O3 sites produces an increase of the isomer shift from $0.13 \mathrm{~mm} / \mathrm{s}$ (without vacancies) up to $0.48,0.32$, and $0.20 \mathrm{~mm} / \mathrm{s}$, respectively (see Table 2). The values of the $I S$ and the $\Delta Q$ obtained with an oxygen vacancy at $\mathrm{O} 1$ site are close to the parameters that characterize the interaction I1 and those obtained with vacancy at $\mathrm{O} 2$ site near the ones of $\mathrm{I} 2$. Finally, for the configuration with a vacancy at $\mathrm{O} 1$ site (associated with the Mössbauer interaction with higher relative fraction, I1) the structure is in good agreement with the distances obtained from EXAFS (1.985 $\AA$, ref 38 ). As a conclusion of this section, the comparison between theory and experiments supports the idea that the two main interactions (I1 and I2) observed in Mössbauer experiments can be associated with $\mathrm{Fe}$ ions substitutionally located at the cationic sites and with oxygen coordination number smaller than six, which is in agreement with EXAFS results.

4.3. System with One Interstitial Fe $\left(\mathrm{Sn}_{16} \mathrm{O}_{32}+\right.$ Interstitial Fe). Some Mössbauer spectra reveal the existence of an interaction (I4) characterized by large isomer shift (IS, on the order of $0.9 \mathrm{~mm} / \mathrm{s})$ and quadrupole splitting $(\Delta Q)$ of around $2.0 \mathrm{~mm} / \mathrm{s}$. This additional interaction appears only in samples prepared by high-energy methods like ball-milling and ion implantation. ${ }^{27,29}$ Since none of the previous calculations predict a high isomer shift, it is considered that this interaction may correspond to interstitial $\mathrm{Fe}$ atoms.

To study this problem, we first verified that the most stable position for an interstitial $\mathrm{Sn}$ or $\mathrm{Fe}$ atom is the octahedral site at the center of the (010) face (the atomic position is $0 ; 1 / 2 ; 1 / 2$ in the unit cell). In this position, an interstitial atom has six ONN: two at $1.71 \AA$ and the other four at $2.34 \AA$. After the structural relaxation, the closest $\mathrm{O}$ atoms relax outward from the interstitial site by $0.18 \AA$ while the other four $\mathrm{O}$ atoms are pulled in toward the interstitial $\mathrm{Fe}$ site by $0.35 \AA$. The equilibrium interstitial $\mathrm{Fe}-\mathrm{ONN}$ bond lengths are then very similar to those corresponding to $\mathrm{Fe}$ in the substitutional site without oxygen vacancies. The formation energy of an interstitial $\mathrm{Fe}$ is in the order of $6.0 \mathrm{eV}$, by $3.0 \mathrm{eV}$ larger than for the substitutional position. Finally, our calculations predict a null magnetic moment at the $\mathrm{Fe}$ interstitial sites.

In the equilibrium structure, the calculated $I S$ and $\Delta Q$ at the Fe site are 0.61 and $0.82 \mathrm{~mm} / \mathrm{s}$, respectively, both smaller than those corresponding to I4. From the study on the role of oxygen vacancies it is found that the preferential site for an oxygen vacancy is close to the interstitial Fe ion. Similar to the substitutional case, the presence of the oxygen vacancies reduces the energy necessary to locate $\mathrm{Fe}$ in the interstitial site, but this energy is also larger than that for the substitutional Fe. Depending on the location of the vacancy, a magnetic moment may appear. This magnetic moment is mainly originated by the polarization of $\mathrm{Fe}$ interstitial atoms. Concerning the $I S$, when vacancies are present, the $I S$ increases up to $0.70 \mathrm{~mm} / \mathrm{s}$ (see Table 3). Although the IS corresponding to an interstitial iron

Table 3. Calculated Hyperfine Parameters $\Delta Q$ and $I S$ at an Interstitial Fe Atom with and without Oxygen Vacancies ${ }^{a}$

\begin{tabular}{ccccc} 
& \multicolumn{4}{c}{$\mathrm{Sn}_{16} \mathrm{O}_{32}+$ interstitial Fe } \\
\cline { 2 - 5 } & $\begin{array}{c}\text { without } \\
\text { vacancy }\end{array}$ & $\begin{array}{c}\text { vacancy at site } \\
\text { O1 vacancy at site }\end{array}$ & $\begin{array}{c}\text { vacancy at site } \\
\text { O3 }\end{array}$ \\
$\begin{array}{c}|\Delta Q| \\
(\mathrm{mm} / \mathrm{s})\end{array}$ & 0.82 & 0.18 & 0.56 & 0.84 \\
$I S(\mathrm{~mm} / \mathrm{s})$ & +0.61 & +0.48 & +0.71 & +0.58
\end{tabular}

${ }^{a} \mathrm{O} 1$ and $\mathrm{O} 2$ correspond to oxygen vacancies in the first shell of oxygen neighbors of $\mathrm{Fe}$, and $\mathrm{O} 3$ is an oxygen vacancy in the second shell of $\mathrm{Fe}$-oxygen neighbors.

is higher than the corresponding one of the substitutional case, the calculated $\Delta Q$ is far away from the experimental one that characterizes I4 $(0.90-1.0 \mathrm{~mm} / \mathrm{s})$. Therefore, to assign this particular interaction, more studies are needed considering different configurations of substitutional and interstitial $\mathrm{Fe}$ and oxygen vacancies.

4.4. Systems with Two Substitutional Fe Atoms $\left(\mathrm{Sn}_{14} \mathrm{Fe}_{2} \mathrm{O}_{32}\right)$. In the previous sections, only one $\mathrm{Fe}$ atom per unit cell was considered, so that the Fe spins were forced to be ferromagnetically aligned. To study the case of an antiferromagnetic (AF) alignment between two $\mathrm{Fe}$ atoms, we performed calculations with two $\mathrm{Fe}$ atoms, initially without oxygen vacancies, for different $\mathrm{Fe}$ atoms distribution. The purpose is to find the preferred configurations and the dependence of the magnetic moments on the $\mathrm{Fe}$ distribution. Figure 3 shows the three $\mathrm{Fe}-\mathrm{Fe}$ configurations studied. The other possible configurations in the SC with larger $\mathrm{Fe}-\mathrm{Fe}$ separations were not considered.

Our results show that the lowest energy is obtained when the Fe atoms are located at the shortest possible distance from each other. This corresponds to $\mathrm{Fe}$ atoms located along the $c$-axis of the rutile structure (configuration $a$, Figure 3 ). Table 4 shows the energies for the FM and AF cases and the magnetic moments of each configuration. As seen in Table 4, for two of the three configurations the most stable phase is the antiferromagnetic one. In consequence, the possibility to obtain a stable ferromagnetic semiconductor with a large magnetic moment depends on the distribution of the $\mathrm{Fe}$ atoms in the $\mathrm{SnO}_{2}$ lattice.

When two $\mathrm{Fe}$ atoms are considered, the $I S$ values at the $\mathrm{Fe}$ sites are on the order of $0.15 \mathrm{~mm} / \mathrm{s}$ for all the configurations 


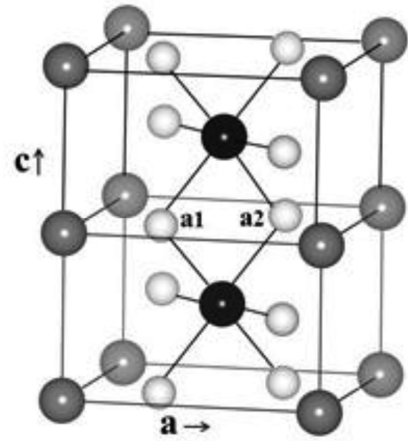

Configuration a

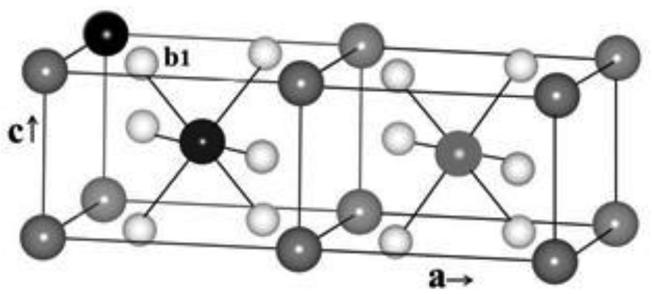

Configuration $\mathrm{b}$

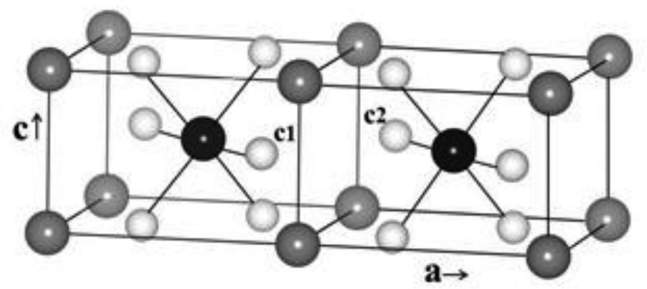

Configuration $\mathrm{c}$

Figure 3. Part of the $2 \times 2 \times 2$ SC showing the different distribution of the Fe atoms (black balls) substituted at Sn sites.

studied. These values are similar to that for one isolated $\mathrm{Fe}$ atoms and in poor agreement with the experimental results. This discrepancy was expected as we were not including oxygen vacancies here. The oxygen vacancies will be considered in the next section.

4.5. Systems with Two Substitutional Fe and Oxygen Vacancies $\left(\mathrm{Sn}_{14} \mathrm{Fe}_{2} \mathrm{O}_{32-\delta}\right)$. It is very difficult to determine experimentally the location of oxygen vacancies, and the computational modeling is required. The study of the magnetic behavior when two $\mathrm{Fe}$ atoms and oxygen vacancies are introduced is far more complicated than in the previous cases since the concentration and distribution of $\mathrm{Fe}$ atoms and vacancies will affect the magnetic interactions. The magnetic and hyperfine properties of the material will be an average of different Fe-defect configurations.

We considered the three configurations shown in Figure 3 with one or two oxygen vacancies. Configuration a has the two Fe ions linked by two $\mathrm{Fe}-\mathrm{O}-\mathrm{Fe}$ paths $(d(\mathrm{Fe}-\mathrm{Fe})=3.19 \AA$ in the unrelaxed structure). Configuration $\mathrm{b}$ has the two Fe linked by only one $\mathrm{Fe}-\mathrm{O}-\mathrm{Fe}$ path $(d(\mathrm{Fe}-\mathrm{Fe})=3.70 \AA$ in the unrelaxed structure). Configuration $\mathrm{c}$ has the $\mathrm{Fe}$ atoms linked by three separate $\mathrm{Fe}-\mathrm{O}-\mathrm{Sn}-\mathrm{O}-\mathrm{Fe}$ chains $(d(\mathrm{Fe}-\mathrm{Fe})=4.74$ $\AA$ ). It is found that one oxygen vacancy in configuration a prefers to be in one of the two $\mathrm{Fe}-\mathrm{O}-\mathrm{Fe}$ paths (sites a1 or a2 in Figure 3) and two oxygen vacancies prefer to occupy both $\mathrm{Fe}-\mathrm{O}-\mathrm{Fe}$ paths. In configuration $\mathrm{b}$ an oxygen vacancy prefers to be in the $\mathrm{Fe}-\mathrm{O}-\mathrm{Fe}$ path (site b1 in Figure 3), and if there are two oxygen vacancies, one prefers to be in the $\mathrm{Fe}-\mathrm{O}-\mathrm{Fe}$ path and the other as a nearest neighbor of one of the $\mathrm{Fe}$ atoms. Finally, for configuration c, the oxygen vacancy prefers to be near one of the $\mathrm{Fe}$ atoms. If a second oxygen vacancy is present, the stable configuration is obtained when the oxygen vacancies are located as a nearest neighbor of each $\mathrm{Fe}$ atom.

Energetically, if a vacancy is located in sites a1 or a2, it is estimated that $\Delta E=E^{\text {anti }}-E^{\text {ferro }}=-0.1 \mathrm{eV}$. Therefore, the lowest energy configuration is the antiferromagnetic one, but the energy difference between the ferromagnetic and antiferromagnetic states is smaller than those of the system without oxygen vacancies. If there are two vacancies in al and a2, the lowest energy clearly corresponds to a ferromagnetic state (see Table 4). Another ferromagnetic state was obtained for one vacancy at site b1. Configuration $c$ with one or two oxygen vacancies gives ferromagnetic alignment with $\Delta E$ higher than that of the configuration without vacancies.

As is shown in Table 4, the magnetic moments at the Fe sites increase when one or two oxygen vacancies are present. We conclude that oxygen vacancies play an important role in the magnetic properties, enhancing the magnetic moments and favoring the ferromagnetic coupling between $\mathrm{Fe}$ atoms.

Regarding the hyperfine parameters, we find that the IS values increase in the presence of oxygen vacancies. In configuration a, two $\mathrm{Fe}$ atoms share two oxygen atoms (a1 and 22 in Figure 3, configuration a) of the rectangular basal plane of their coordination octahedra (O1 site). If only one of these oxygen atoms is removed, the resulting $I S$ and $\Delta Q$ are

Table 4. Calculated Hyperfine Parameters $\Delta Q$ and $I S$ at an Fe Impurity, Difference between the Energies $\left(\Delta E=E^{\text {anti }}-E^{\text {ferro }}\right)$, and Magnetic Moments in the SC $\left(\mu^{\mathrm{SC}}\right)$ for the Different Vacancy Positions Studied When There Are Two Substitutional Fe Atoms in the $\mathrm{SC}^{a}$

\begin{tabular}{|c|c|c|c|c|c|c|c|}
\hline configuration & & $I S(\mathrm{~mm} / \mathrm{s})$ & $|\Delta Q|(\mathrm{mm} / \mathrm{s})$ & $\mu^{\mathrm{imp}}\left(\mu_{\mathrm{B}}\right)$ & $\mu^{\mathrm{SC}}\left(\mu_{\mathrm{B}}\right)$ & $\Delta E(\mathrm{eV})$ & magnetic order \\
\hline \multirow[t]{3}{*}{$a$} & NV & 0.15 & 1.26 & \pm 1.80 & 0.0 & -0.136 & $\mathrm{AF}$ \\
\hline & $1 \mathrm{~V}$ & 0.38 & 0.65 & \pm 3.52 & 0 & -0.095 & $\mathrm{AF}$ \\
\hline & $2 \mathrm{~V}$ & 0.50 & 0.65 & 3.40 & 8.5 & 0.082 & FM \\
\hline \multirow[t]{2}{*}{$b$} & $\mathrm{NV}$ & 0.14 & 2.50 & 1.63 & 0.0 & -0.068 & $\mathrm{AF}$ \\
\hline & $1 \mathrm{~V}$ & $0.32 / 0.31$ & $1.30 / 2.02$ & $2.43 / 2.48$ & 6.0 & 0.054 & FM \\
\hline \multirow[t]{2}{*}{$c$} & $\mathrm{NV}$ & 0.12 & 2.24 & 1.63 & 4.0 & 0.013 & FM \\
\hline & $2 \mathrm{~V}$ & 0.32 & 1.34 & 2.38 & 6.0 & 0.027 & FM \\
\hline
\end{tabular}

${ }^{a} \mathrm{NV}, 1 \mathrm{~V}$, and $2 \mathrm{~V}$ indicate the cases without oxygen vacancies or one or two oxygen vacancies in the SC. In the case of Configuration $b$ with one oxygen vacancy the results for the IS, the $|\Delta Q|$, and $\mu^{\text {imp }}$ refers to each inequivalent Fe site of the structure. 
close to experimental ones corresponding to I1. If two oxygen atoms are removed, a further increment of IS is found. In configuration $b$, the two $\mathrm{Fe}$ atoms share one oxygen atom (b1 in Figure 3, configuration b) that is of type $\mathrm{O} 1$ for the Fe site placed in the center of the unit cell and is apical oxygen $\mathrm{O} 2$ for the $\mathrm{Fe}$ atom located at a vertex of the unit cell. Removing this shared oxygen atom, the two nonequivalent iron atoms remain with almost the same IS and with different $\Delta Q$ (see Table 4). The average values of $I S$ and $\Delta Q$ for these irons are in very good agreement with the experimental hyperfine parameters of I2. In configuration $\mathrm{c}$, the two $\mathrm{Fe}$ atoms do not share oxygen nearest neighbors. The oxygen atoms labeled $\mathrm{c} 1$ and $\mathrm{c} 2$ in Figure 3 (configuration c) are at the apical (O2) position of two different $\mathrm{Fe}$ octahedral coordination. If both atoms are removed, the $I S$ and $\Delta Q$ are in the range of experimental hyperfine parameters of $\mathrm{I} 2$.

The calculated values of the $I S$ and $\Delta Q$ for the substitutional cases with one and two oxygen vacancies are included in Figure 1 (open symbols). If an apical oxygen $\mathrm{O} 2$ atom is removed, the IS and $\Delta Q$ have values close to experimental ones corresponding to I2, whether the iron atom has or does not have another Fe atom as nearest neighbor (configuration b or c, blue symbols in Figure 1). In the cases where oxygen $\mathrm{O} 1$ is removed, the calculated hyperfine parameters (red symbols in Figure 1) are close to the experimental values corresponding to I1.

4.6. Systems with One Interstitial and One Substitutional Fe Atom. We consider the interstitial and susbtitutional $\mathrm{Fe}$ atoms with and without oxygen vacancies. The two interstitial + substitutional configurations considered here are (a) An interstitial Fe atom (Fe(int)) located at position (0, 1/2, $1 / 2)$ and the substitutional $\mathrm{Fe}$ atom ( $\mathrm{Fe}$ (subst)) located at (1/ $2,1 / 2,1 / 2)$ and (b) Fe(int) located at $(0,1 / 2,1 / 2)$ and the $\mathrm{Fe}$ (subst) at $(0,0,0)$. These configurations correspond to the smaller $\mathrm{Fe}$ (subst)-Fe(int) separation distances. The predicted $I S$ and $\Delta Q$ values are shown in Table 5 . When one oxygen

Table 5. Calculated Hyperfine Parameters $\Delta Q$ and IS When There Are Two Fe Atoms, an Interstitial One and a Substitutional One, Difference between the Energies $(\Delta E=$ $\left.E^{\text {anti }}-E^{\text {ferro }}\right)$, and Magnetic Moment in the SC $\left(\mu^{\mathrm{SC}}\right)$

\begin{tabular}{cllcccc} 
configuration & & & $\begin{array}{c}I S \\
(\mathrm{~mm} / \mathrm{s})\end{array}$ & $\begin{array}{c}|\Delta \mathrm{Q}| \\
(\mathrm{mm} / \mathrm{s})\end{array}$ & $\begin{array}{c}\mu^{\mathrm{imp}} \\
\left(\mu_{\mathrm{B}}\right)\end{array}$ & $\begin{array}{c}\mu^{\mathrm{SC}} \\
\left(\mu_{\mathrm{B}}\right)\end{array}$ \\
\hline$a$ & $\mathrm{NV}$ & Int & 0.50 & 1.43 & +0.36 & -0.76 \\
& & Sus & 0.45 & 0.73 & -0.66 & \\
& \multirow{2}{*}{$\mathrm{V}$} & Int & 0.40 & 0.88 & -0.17 & -3.77 \\
& & Sus & 0.52 & 0.64 & +3.45 & \\
& $\mathrm{NV}$ & Int & 0.65 & 0.72 & -1.69 & -2.56 \\
& & Sus & 0.68 & 0.42 & -3.63 & \\
& \multirow{2}{*}{$\mathrm{IV}$} & Int & 0.46 & 1.12 & +0.95 & -2.00 \\
& & Sus & 0.60 & 1.95 & -2.56 & \\
\hline
\end{tabular}

vacancy is introduced, the oxygen vacancy prefers to be located as a nearest neighbor of both Fe atoms. In this situation, the IS for $\mathrm{Fe}$ (subst) is similar to those of the experimentally observed interactions $\mathrm{I} 1$ and $\mathrm{I} 2$.

In ref 30, the authors analyze ${ }^{57} \mathrm{Mn} \rightarrow{ }^{57} \mathrm{Fe}$ implanted in $\mathrm{SnO}_{2}$ films and report that the Mössbauer spectra presents two doublets, one with $I S=0.48 \mathrm{~mm} / \mathrm{s}$ and $\Delta Q=0.91 \mathrm{~mm} / \mathrm{s}$ and the other with $I S=0.89 \mathrm{~mm} / \mathrm{s}$ and $\Delta Q=2.20 \mathrm{~mm} / \mathrm{s}$. Comparing with Table 3 (calculated values for one interstitial $\mathrm{Fe}$ atom), there is poor agreement between experimental and calculated results, but there is good agreement with the situation where the interstitial $\mathrm{Fe}$ has a susbtitutional iron near neighbor (see Table 5, configuration $b$ with one oxygen vacancy).

\section{LONG-RANGE FERROMAGNETIC ORDER}

In section 4.5 we discussed on the effect of oxygen vacancies on the magnetic alignment considering two $\mathrm{Fe}$ atoms and one or two oxygen vacancies. We showed that the presence of one or two oxygen vacancies in the $\mathrm{Fe}-\mathrm{O}-\mathrm{Fe}$ paths clearly favors ferromagnetic ordering. Such an ferromagnetic entity (the $\mathrm{Fe}$ atoms and oxygen vacancy) was described as a bound magnetic polaron (BMP). In order to explore the long-range ferromagnetic order, we considered two BMPs inside a larger SC of 108 atoms $(3 a \times 2 a \times 3 c$ SC, see Figure $4 a)$. The
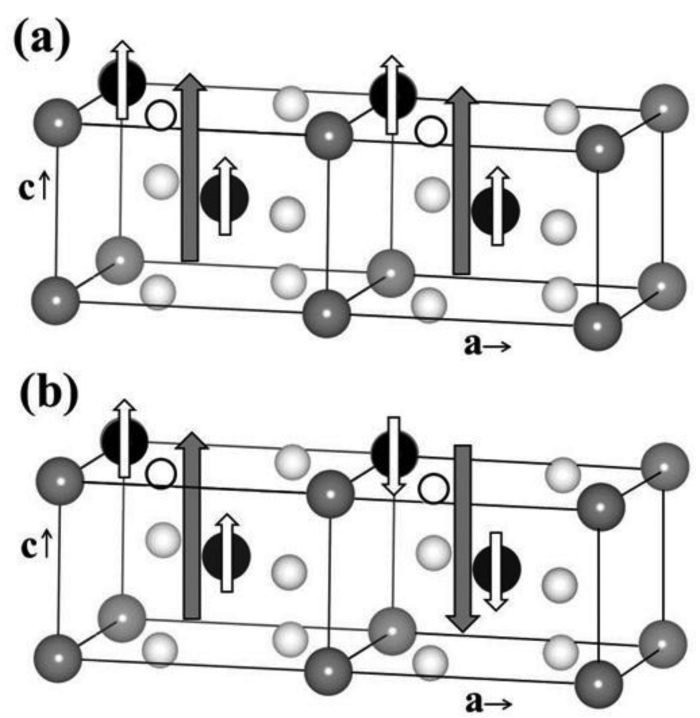

Figure 4. Part of the $3 \times 2 \times 3$ SC showing the bound magnetic polaron model for ferromagnetic alignment (a) and antiferromagnetic alignment (b) among BMPs. Open circles indicates the position of the oxygen vacancies.

distance between the BMPs is $4.74 \AA$. Note that the BMP corresponds to two $\mathrm{Fe}$ atoms in configuration $\mathrm{b}$ with the oxygen vacancy in the position b1. Two possible BMP alignments were considered: ferromagnetic (Figure 4a) or antiferromagnetic (Figure $4 \mathrm{~b}$ ). It is found that the energy difference between both alignments is $\Delta E=-0.05 \mathrm{eV}$; i.e., the antiferromagnetic alignment is favored. Thus, although an increase in iron concentration would favor a neighboring situation with the increase in the number of BMP, the antiferromagnetic BMP alignment would reduce the magnetization, which is in very good agreement with the experimental results. In consequence, the origin of the ferromagnetic contribution is still unexplained, and another mechanism must be considered to explain the ferromagnetic response of Fe-doped $\mathrm{SnO}_{2}$.

At this point it is important to return to the experiments. It was reported that the codoping of $\mathrm{SnO}_{2}$ with $\mathrm{Fe}$ and $\mathrm{Sb}^{5+}$ ions increases the saturation magnetization. ${ }^{39}$ This experimental result led us to study the effect of extra electron doping on the ferromagnetic alignment. The electron doping was simulated in two different ways: adding one electron to the 108-atom SC or replacing one indigenous $\mathrm{Sn}$ atom by a donor impurity. For both approaches we found that the ferromagnetic coupling 
between $\mathrm{BMP}$ is the most stable configuration with $\Delta E=E^{\text {anti }}$ $-E^{\text {ferro }}$ on the order of $+0.3 \mathrm{eV}$. A Bader analysis reveals that $20 \%$ of the added charge is localized in the four $\mathrm{Fe}$ atomic spheres and the remaining in the interstitial region. This result shows that electron doping plays a fundamental role in mediating the nonlocal magnetic coupling between two BMP.

\section{CONCLUSIONS}

We have shown here a theoretical investigation of Fe-doped rutile $\mathrm{SnO}_{2}$. Our calculations, assuming different structural scenarios, allow us to assign the two main interactions (I1 and I2) observed in the Mössbauer experiments reported in the literature to $\mathrm{Fe}^{3+}$ replacing $\mathrm{Sn}^{4+}$ in the rutile structure with oxygen vacancies close to the dopant. Other interactions were found in some experiments. These additional interactions have lower intensity than I1 and I2. The third interaction (I3) is characterized by a low IS (on the order of $0.20 \mathrm{~mm} / \mathrm{s}$ ). Based on the low IS it can be assigned, in principle, to $\mathrm{Fe}$ atoms replacing $S n$ with an oxygen vacancy far away from the first shell of oxygen neighbors. The interaction I4, characterized by a high $I S$ value (on the order of $1.0 \mathrm{~mm} / \mathrm{s}$ ), can be tentatively assigned to $\mathrm{Fe}$ ions located at interstitial sites with iron and oxygen vacancies in its close vicinity. The fact that the energy necessary to locate an iron atom at an interstitial site is large compared with the substitutional configuration allows us to understand why this hyperfine interaction is only observed in samples obtained by high-energy methods such as ion implantation or high-energy ball milling. From our results it is clear that the overall hyperfine properties of Fe-doped $\mathrm{SnO}_{2}$ are a statistical average of different defect configuration. In order to reach a better understanding of the interactions I3 and I4, different configurations (considering different concentrations and distributions of $\mathrm{Fe}$ atoms and oxygen vacancies) must be considered.

An important result of our calculations is that doping lowers the formation energy of vacancies, so that doped systems will have more vacancies than the undoped ones. The strong interaction between oxygen vacancies and $\mathrm{Fe}$ atoms increases the local magnetic moment of the Fe atom. We also found that the oxygen vacancies enhance the ferromagnetic coupling between $\mathrm{Fe}$ atoms. Fe ions are coupled ferromagnetically when they are mediated by an oxygen vacancy forming a BMP. In cases where there are no free carriers, BMPs are coupled antiferromagnetically. Carrier doping produces the ferromagnetic alignment between BMPs. Therefore, oxygen vacancies would be responsible for forming BMPs and free carriers for inducing the ferromagnetic coupling between BMPs.

The overall magnetic properties of Fe-doped rutile $\mathrm{SnO}_{2}$ are an average over many possible defect configurations, and therefore ferromagnetism in this system is a complex phenomenon, which involves the interplay between oxygen vacancies and doping atoms. Our results support the hypothesis that oxygen vacancies play an important role in the magnetic properties of $\mathrm{Fe}$-doped rutile $\mathrm{SnO}_{2}$ and can explain the diversity of magnetic behaviors experimentally observed for samples grown in different conditions. Similar conclusions were obtained in the case of $\mathrm{Fe}$-doped rutile $\mathrm{TiO}_{2},{ }^{35} \mathrm{Zn}$-ferrite, ${ }^{40}$ and other doped metallic oxides such as Fe-doped $\operatorname{In}_{2} \mathrm{O}_{3}$ and Co-doped $\mathrm{ZnO}^{41}$ In all these systems it was demonstrated that oxygen vacancies in the $\mathrm{Fe}-\mathrm{O}-\mathrm{Fe}$ (or $\mathrm{Co}-\mathrm{O}-\mathrm{Co}$ ) paths clearly favor ferromagnetic ordering.

\section{AUTHOR INFORMATION}

\section{Corresponding Author}

*E-mail mudarra@fisica.unlp.edu.ar; Tel 54-221-4230122/ 4247201/4246062 (A.M.M.N.).

\section{Notes}

The authors declare no competing financial interest.

\section{ACKNOWLEDGMENTS}

This work was partially supported by CONICET (Grants PIP6005, PIP01111, and PIP0002), National University of La Plata (Grants X543 and 11/X708), ANPCyT (Grants PICT 00898 and PICT-2013-2616), and MINCyT-DAAD (Grant DA13/02). Calculations wrere performed using the Huge Cluster, University of Aarhus, Denmark.

\section{REFERENCES}

(1) Batzill, M.; Diebold, U. The Surface and Materials Science of Tin Oxide. Prog. Surf. Sci. 2005, 79, 47-154.

(2) Göpel, W.; Schierbaum, K. D. $\mathrm{SnO}_{2}$ Sensors: Current Status and Future Prospects. Sens. Actuators, B 1995, 26, 1-12.

(3) Das, S.; Jayaraman, V. $\mathrm{SnO}_{2}: \mathrm{A}$ Comprehensive Review on Structures and Gas Sensors. Prog. Mater. Sci. 2014, 66, 112-255.

(4) Punnoose, A.; Hays, J.; Thurber, A.; Engelhard, M. H.; Kukkadapu, R. K.; Wang, C.; Shutthanandan, V.; Thevuthasan, S. Development of High-Temperature Ferromagnetism in $\mathrm{SnO}_{2}$ and Paramagnetism in SnO by Fe Doping. Phys. Rev. B 2005, 72, 054402.

(5) Punnoose, A.; Hays, J.; Gopal, V.; Shutthanandan, V. RoomTemperature Ferromagnetism in Chemically Synthesized $\mathrm{Sn}_{1-\mathrm{x}} \mathrm{Co}_{\mathrm{x}} \mathrm{O}_{2}$ Powders. Appl. Phys. Lett. 2005, 85, 1559-1561.

(6) Coey, J. M. D.; Douvalis, A. P.; Fitzgerald, C. B.; Venkatesan, M. Ferromagnetism in Fe-Doped $\mathrm{SnO}_{2}$ Thin Films. Appl. Phys. Lett. 2004, $84,1332-1334$.

(7) Fitzgerald, C. B.; Venkatesan, M.; Douvalis, A. P.; Huber, S.; Coey, J. M. D. $\mathrm{SnO}_{2}$ Doped with $\mathrm{Mn}, \mathrm{Fe}$ or Co: Room Temperature Dilute Magnetic Semiconductors. J. Appl. Phys. 2004, 95, 7390-7392.

(8) Nomura, K.; Barrero, C. A.; Sakuma, J.; Takeda, M. RoomTemperature Ferromagnetism of Sol-Gel-Synthesized $\mathrm{Sn}_{1-\mathrm{x}}{ }^{57} \mathrm{Fe}_{\mathrm{x}} \mathrm{O}_{2-\delta}$ Powders. Phys. Rev. B 2007, 75, 184411.

(9) Rodríguez Torres, C. E.; Errico, L. A.; Golmar, F.; Navarro Mudarra, A. M.; Cabrera, A. F.; Duhalde, S.; Sánchez, F. H.; Weissmann, M. The Role of the Dopant in the Magnetism of FeDoped $\mathrm{SnO}_{2}$ Films. J. Magn. Magn. Mater. 2007, 316, e219-e222.

(10) Srivastava, S. K.; Lejay, P.; Barbara, B.; Pailhès, S.; Madigou, V.; Bouzerar, G. Possible Room-Temperature Ferromagnetism in KDoped $\mathrm{SnO}_{2}$ : X-Ray Diffraction and High-Resolution Transmission Electron Microscopy Study. Phys. Rev. B 2010, 82, 193203.

(11) Espinosa, A.; García-Hernández, M.; Menéndez, N.; Prieto, C.; de Andrés, A. Ferromagnetism in $\mathrm{SnO}_{2}$-Based Multilayers: Clustering of Defects Induced by Doping. Phys. Rev. B 2010, 81, 064419.

(12) Hong, N. H.; Poirot, N.; Sakai, J. Ferromagnetism Observed in Pristine $\mathrm{SnO}_{2}$ Thin Films. Phys. Rev. B 2008, 77, 033205.

(13) Chang, G. S.; Forrest, J.; Kurmaev, E. Z.; Morozovska, A. N.; Glinchuk, M. D.; McLeod, J. A.; Moewes, A.; Surkova, T. P.; Nguyen Hoa, H. Oxygen-Vacancy-Induced Ferromagnetism in Undoped $\mathrm{SnO}_{2}$ Thin Films. Phys. Rev. B 2012, 85, 165319.

(14) Mudarra Navarro, A.; Errico, L. A.; Rodriguez Torres, C.; Cabrera, A. F.; Bilovol, V.; Rentería, M.; Weissmann, M. In Rutile: Properties, Synthesis and Applications; Nova Science: Hauppauge, NY, 2012.

(15) Khalid, D. M.; Setzer, A.; Ziese, M.; Esquinazi, P.; Spemann, D.; Pöppl, A.; Goering, E. Ubiquity of Ferromagnetic Signals in Common Diamagnetic Oxide Crystals. Phys. Rev. B 2010, 81, 214414.

(16) Coey, J. M. D.; Stamenov, P.; Gunning, R. D.; Venkatesan, M.; Paul, K. Ferromagnetism in Defect-Ridden Oxides and Related Materials. New J. Phys. 2010, 12, 053025. 
(17) Bolzan, A.; Fong, C.; Kennedy, B.; Howard, C. J. Structural Studies of Rutile-Type Metal Dioxides. Acta Crystallogr., Sect. B 1997, 53, 373-380.

(18) Blaha, P.; Schwarz, K.; Madsen, G.; Kvasnicka, D.; Luitz, J. WIEN2k, An Augmented Plane Wave Plus Local Orbitals Program for Calculating Crystal Properties; Technical Universität Wien: Austria, 1999.

(19) Sjöstedt, E.; Nordström, L.; Singh, D. J. An Alternative Way of Linearizing the Augmented Plane-Wave Method. Solid State Commun. 2000, 114, 15-20.

(20) Madsen, G. K. H.; Blaha, P.; Schwarz, K.; Sjöstedt, E.; Nordström, L. Efficient Linearization of the Augmented Plane-Wave Method. Phys. Rev. B 2001, 64, 195134.

(21) Duhalde, S.; Vignolo, M. F.; Golmar, F.; Chiliotte, C.; Rodríguez Torres, C. E.; Errico, L. A.; Cabrera, A. F.; Rentería, M.; Sánchez, F. H.; Weissmann, M. Appearance of Room-Temperature Ferromagnetism in Cu-Doped $\mathrm{TiO}_{2-\delta}$ Films. Phys. Rev. B 2005, 72, 161313(R).

(22) Perdew, J. P.; Wang, Y. Accurate and Simple Analytic Representation of the Electron-Gas Correlation Energy. Phys. Rev. B 1992, 45, 13244-13249.

(23) Anisimov, V. I.; Solovyev, I. V.; Korotin, M. A.; Czyzyk, M. T.; Sawatzky, G. A. Density-Functional Theory and NiO Potoemission Spectra. Phys. Rev. B 1993, 48, 16929-16934.

(24) Zboril, R.; Mashlan, M.; Petridis, D. Iron(III) Oxides from Thermal Processess- Synthesis, Structural and Magnetic Properties, Mössbauer Spectroscopy Characterization, and Applications. Chem. Mater. 2002, 14, 969-982.

(25) Sorescu, M.; Diamandescu, L.; Tarabasanu-Mihaila, D.; Teodorescu, V. S.; Howard, B. H. Hydrothermal Synthesis and Structural Characterization of $(1-\mathrm{x}) \alpha-\mathrm{Fe}_{2} \mathrm{O}_{3}-\mathrm{x} \mathrm{SnO}_{2}$ Nanoparticles. J. Phys. Chem. Solids 2004, 65, 1021-1029.

(26) Liu, J. F.; Lu, M. F.; Chai, P.; Fu, L.; Wang, Z. L.; Cao, X. Q.; Meng, J. The Magnetic and Structural Properties of HydrothermalSynthesized Single-Crystal $\mathrm{Sn}_{1-\mathrm{x}} \mathrm{Fe}_{\mathrm{x}} \mathrm{O}_{2}$ Nanograins. J. Magn. Magn. Mater. 2007, 317, 1-7.

(27) Bilovol, V.; Mudarra Navarro, A. M.; Rodríguez Torres, C. E.; Sánchez, F. H.; Cabrera, A. F. Magnetic and Structural Study of $\mathrm{Fe}$ Doped Tin Dioxide. Physica B 2009, 404, 2834-2837.

(28) Nomura, K.; Barrero, C. A.; Kuwano, K.; Yamada, Y.; Saito, T.; Kuzmann, E. ${ }^{57} \mathrm{Fe}$ Mossbauer Study of $\mathrm{Fe}$ and $\mathrm{Sb}$ Doped $\mathrm{SnO}_{2}$ Powders. Hyperfine Interact. 2009, 191, 25-32.

(29) Nomura, K.; Reuther, H. Nano-Structure Analysis of $\mathrm{Fe}$ Implanted $\mathrm{SnO}_{2}$ Films by ${ }^{57} \mathrm{Fe}$ and ${ }^{119} \mathrm{Sn}$ CEMS. Hyperfine Interact. 2009, 191, 159-165.

(30) Gunnlaugsson, H. P.; Nomura, K.; Mølholt, T. E.; Shayestehaminzadeh, S.; Johnston, K.; Mantovan, R.; Masenda, H.; Ncube, M.; Bharuth-Ram, K.; Gislason, H.; Langouche, G.; Naidoo, D.; Ólafsson, S.; Weyer, G. Characterization of Fe States in Dilute ${ }^{57} \mathrm{Mn}$ Implanted $\mathrm{SnO}_{2}$ Film. Hyperfine Interact. 2014, 226, 389-396.

(31) Shirane, G.; Cox, D. E.; Ruby, S. L. Mössbauer Study of Isomer Shift, Quadrupole Interaction, and Hyperfine Field in Several Oxides Containing ${ }^{57} \mathrm{Fe}$. Phys. Rev. 1962, 125, 1158-1165.

(32) Eriksson, O.; Svane, A. Isomer Shifts and Hyperfine Fields in Iron Compounds. J. Phys.: Condens. Matter 1989, 1, 1589-1599.

(33) Mudarra Navarro, A. M.; Rodríguez Torres, C.; Errico, L. A. Calibration of the Isomer Shift of the $14.4 \mathrm{keV}$ Transition of ${ }^{57} \mathrm{Fe}$. Hyperfine Interact. 2011, 202, 117-121.

(34) Dufeck, P.; Blaha, P.; Schwarz, K. Determination of the Nuclear Quadrupole Moment of ${ }^{57} \mathrm{Fe}$. Phys. Rev. Lett. 1995, 75, 3545-3548.

(35) Mudarra Navarro, A. M.; Rodríguez Torres, C. E.; Bilovol, V.; Cabrera, A. F.; Errico, L. A.; Weissmann, M. Study of the Relation Between Oxygen Vacancies and Ferromagnetism in Fe-Doped $\mathrm{TiO}_{2}$ Nano-powders. J. Appl. Phys. 2014, 115, 223908.

(36) Errico, L. A.; Weissmann, M.; Rentería, M. Theoretical Study of Magnetism in Transition-Metal-Doped $\mathrm{TiO}_{2}$ And $\mathrm{TiO}_{2-\delta}$. Phys. Rev. $B$ 2005, 72, 184425 .

(37) Rodríguez Torres, C. E.; Cabrera, A. F.; Errico, L. A.; Adán, C.; Requejo, F. G.; Weissmann, M.; Stewart, S. J. Local Structure and Magnetic Behaviour of Fe-Doped $\mathrm{TiO}_{2}$ Anatase Nanoparticles:
Experiments and Calculations. J. Phys.: Condens. Matter 2008, 20, 135210.

(38) Bilovol, V.; Mudarra Navarro, A. M.; Rodríguez Torres, C. E.; Cabrera, A. F. Effect of Thermal Treatment in Vacuum on Fe-Doped $\mathrm{SnO}_{2}$ Powders. Physica B 2012, 407, 3214-3217.

(39) Nomura, K.; Shima, A. Dilute Magnetism and Mossbauer Study of Nanoscaled $\mathrm{SnO}_{2} \mathrm{Co}$-Doped with Fe, Co, and $\mathrm{Sb}$, Prepared by SolGel Method. AIP Conf. Proc. 2012, 1489, 13-20.

(40) Rodríguez Torres, C. E.; Pasquevich, G. A.; Mendoza Zélis, P.; Golmar, F.; Heluani, S. P.; Nayak, S. K.; Adeagbo, W. A.; Hergert, W.; Hoffmann, M.; Ernst, A.; Esquinazi, P.; Stewart, S. J. Oxygen-VacancyInduced Local Ferromagnetism as a Driving Mechanism in Enhancing the Magnetic Response of Ferrites. Phys. Rev. B 2014, 89, 104411.

(41) Qi, S.; Jiang, F.; Fan, J.; Wu, H.; Zhang, S. B.; Gehring, G. A.; Zhang, Z.; Xu, X. Carrier-Mediated Nonlocal Ferromagnetic Coupling Between Local Magnetic Polarons in Fe-Doped $\mathrm{In}_{2} \mathrm{O}_{3}$ and Co-Doped ZnO. Phys. Rev. B 2011, 84, 205204. 